\title{
Elementos no frecuentes en cálculos renales
}

\author{
J.L. Rodríguez-Miñón Cifuentes*, E. Salvador***, Mํㅡ. Traba Villameytide**. \\ *Servicio de Urología. **Servicio de Bioquímica. Fundación Jiménez Díaz-UTE. \\ ***Facultad de Geología. Universidad Autónoma de Madrid.
}

Actas Urol Esp 2006; 30 (1): 57-60

\section{RESUMEN}

ELEMENTOS NO FRECUENTES EN CÁLCULOS RENALES

Se analizó un grupo de 54 cálculos expulsados espontáneamente después de padecer un cólico nefrítico. Dos grupos de cálculos fueron encontrados: papilares y no papilares. Todos los cálculos fueron analizados por espectroscopia infrarroja y por microscopia electrónica de barrido y EDAX.

$\mathrm{Al}$ ser analizados los cálculos por EDAX se detectaron los siguientes elementos químicos; $\mathrm{C}, \mathrm{N}, \mathrm{O}$, $\mathrm{Na}, \mathrm{S}, \mathrm{Mg}, \mathrm{Al}, \mathrm{Si}, \mathrm{Cl}, \mathrm{K}, \mathrm{Ca}, \mathrm{Mn}, \mathrm{Fe}, \mathrm{Ni}, \mathrm{Zn}$. El posible origen de estos elementos es analizado en este trabajo.

Palabras clave: Cálculo renal. EDX. Elementos no usuales

\section{ABSTRACT \\ UNUSUAL ELEMENTS IN RENAL CALCULI}

A group of 54 renal calculi were spontaneously passed renal stone after a nephritic colic. Two groups of calculi were found: papillary and non-papillary calculi. All calculi were analyzed by infrared spectroscopy and electronic microscopy scan (EMS) and EDAX.

When the stones were analyzed with EDAX, elements such as $\mathrm{C}, \mathrm{N}, \mathrm{O}, \mathrm{Na}, \mathrm{S}, \mathrm{Mg}, \mathrm{Al}, \mathrm{Si}, \mathrm{Cl}, \mathrm{K}, \mathrm{Ca}$, $\mathrm{Mn}, \mathrm{Fe}, \mathrm{Ni}, \mathrm{Zn}$ were detected. The possible origin of these elements is discussed in this work.

Keywords: Renal calculi. EDAX. Unusual element.

$\mathrm{L}$ os cálculos renales están compuestos por componentes habituales de la orina y/o por componentes no habituales de la misma. Estos últimos se originan por alteraciones metabólicas (como son la xantinuria, que cursa con cálculos de xantina o la cistinuria, que cursa con cálculos de cistina), por la acción de agentes microbianos (dando origen a cálculos de estruvita) o por la administración de fármacos ${ }^{1,2}$.

El estudio de los cálculos renales se basa en un primer reconocimiento microscópico o morfológico que puede darnos una orientación sobre la composición de los mismos ${ }^{3}$.

Las técnicas más utilizadas para el estudio de los cálculos renales son: el análisis químico cualitativo, la espectrometría infrarroja, la espectrometría de masas, el estudio en lámina delgada por microscopia con luz polarizada y en algunos casos se realiza un estudio por microscopia electrónica de barrido y EDAX. Además puede hacerse un análisis por difracción de rayos $\mathrm{X}$ y por cromatografía en capa fina o por cromatografía líquida de alta resolución.

La aplicación del análisis con EDAX permite reconocer elementos químicos no encontrados con otras técnicas anteriormente citadas, como la espectrometría infrarroja.

\section{MATERIALES Y MÉTODOS}

Se analizaron un grupo de cálculos $(n=54)$ eliminados espontáneamente después de un cólico nefrítico. Generalmente, se trata de cálculos papilares con una cara convexa lisa y una cara cóncava irregular que corresponde a la huella de implantación del cálculo con el extremo de la papila. Existe otro tipo de cálculos que no son papilares. 
Los cálculos fueron analizados por espectroscopia infrarroja, mediante el sistema de comprimidos de bromuro potásico y por microscopia electrónica de barrido y EDAX.

\section{RESULTADOS}

El análisis por espectroscopia infrarroja de los 54 cálculos mostró que todos los cálculos estaban constituidos por oxalato cálcico monohidrato (whewellita). También se encontró, en alguno de ellos, carbonato apatita en el lugar de unión con la papila. Dos cálculos presentaban ácido úrico y otro cálculo tenía brushita.

Todos los cálculos fueron sometidos al análisis por microscopia electrónica de barrido y EDAX, detectándose los siguientes elementos poco habituales: Na, Mg, Ni, K, Zn, Bi, S, Al, Ti, Si, Cl, Mn y Fe en los porcentajes de distribución de los cálculos estudiados (Tabla 1).

La concentración de los elementos expresada en \% de número atómico (At), generalmente es muy irregular dentro de los distintas partes del cálculo analizado. Así, tenemos un contenido muy diferente en $\mathrm{Na}$ (25,35 y 7,85 At\%) en el cálculo 821 mientras que encontramos menos diferencias en dos zonas estudiadas en el cálculo 878, siendo su contenido de $\mathrm{Na}$ de 39,78 y 28,88 At\%. Sin embargo, el cálculo 1.413 muestra diferentes concentraciones de $\mathrm{Na}$ según la zona analizada $(1,87 ; 0,54 ; 10,5 ; 0,4$ y 0,02 At\%). El contenido

\section{Tabla 1}

Porcentaje de distribución de los elementos en los 50 cálculos estudiados

\begin{tabular}{cc}
\hline Elemento & Porcentaje \\
\hline $\mathrm{Bi}$ & 0,54 \\
$\mathrm{Zn}$ & 0,54 \\
$\mathrm{Ni}$ & 1,08 \\
$\mathrm{Fe}$ & 2,70 \\
$\mathrm{Mn}$ & 0,54 \\
$\mathrm{Si}$ & 4,86 \\
$\mathrm{Ti}$ & 2,16 \\
$\mathrm{Al}$ & 4,32 \\
$\mathrm{Mg}$ & 5,94 \\
$\mathrm{~S}$ & 18,36 \\
$\mathrm{Na}$ & 10,80 \\
$\mathrm{Cl}$ & 15,12 \\
$\mathrm{~K}$ & 19,98 \\
\hline
\end{tabular}

en Mg en este mismo cálculo oscila, según el área estudiada, entre 1,$24 ; 1,47 ; 24,69 ; 1,52$ y 8,92 At\%. El K varió en el cálculo 804 entre 6,8 y 28,26 At \%t; en el cálculo 833 fue de 1,$71 ; 1,2$; 15,47 y 0,2 At\%. El cálculo 1.365 sólo dio en el área analizada 54,7 At\%. El Ni en el cálculo 1.361 sólo se detectó en un 2,04 At\% mientras que el $\mathrm{Zn}$, en este mismo cálculo, se encontró en un porcentaje de 5,12 At\%. El Bi en el cálculo 1.413 se detectó solamente en una zona del cálculo de las cuatro analizadas y su porcentaje fue de 2,21 At\%. El S se encuentra en muchos de los cálculos analizados. En el cálculo 1.384, prácticamente presenta el mismo porcentaje $(31,99$ y 32,53 At $\%)$ en dos zonas analizadas, mientras que en el cálculo 1.383 da resultados muy diferentes según la parte del cálculo analizada $(15,19 ; 56,25$ At\%) y 0,4; 23,74; 0,2 y 18,23 At\% para el cálculo 1.461. La concentración de $\mathrm{Al}$ en el cálculo 871 es muy diferente según la zona del cálculo analizada $(26,4 ; 4,88 ; 5,22 ; 8,72$ y 0,42 At\%). El Si en el cálculo 842 se encuentra en proporciones iguales en todas las zonas analizadas $(4,85 ; 4,95$; 4,43 At\%) mientras que en el cálculo 871 hay diferencias según las distintas zonas $(42,32$; 8,$75 ; 17,17 ; 20,58$ At\%). En el caso del $\mathrm{Cl}$ se encuentran muchas diferencias según la parte analizada del cálculo: 3,20; 53,5; 29,74; 23,27 y 0 At\%. El cálculo 1.940 dio una alta concentración de Ti en la zona analizada 46,79 At\% mientras que el cálculo 871 dio 1,9, 0 y 0 At \% en las tres zonas analizadas. Un hecho similar sucede con el cálculo 1.413 en el que de las 5 zonas analizadas sólo se encontró Mn en una (3,27 At\%). El Fe presenta muchas diferencias según la zona del cálculo analizada. En el cálculo 871 encontramos 23,$94 ; 6,11 ; 0$ y 0 At\% y en el cálculo 1.413 encontramos que según las zonas analizadas el Fe se encuentra en diferentes porcentajes: 6,78; 1,63; 0,88; 2,26 y 12,88 At\%. Las Figuras 1, 2, 3, $4,5,6$ y 7 recogen el porcentaje de los elementos mas destacados del anagrama obtenido por EDAX.

\section{DISCUSIÓN}

Gran parte de los elementos presentados en este trabajo son constituyentes naturales de los cálculos renales y forman parte del individuo. Otros, sin embargo, proceden de la ingesta de 


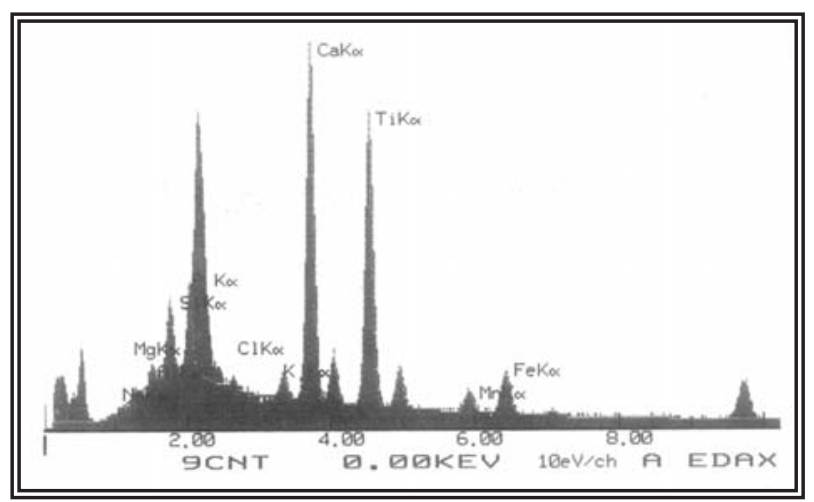

FIGURA 1. Contenido de iones (\% At). Na: 0,$4 ; \mathrm{Mg}: 1,24 ; \mathrm{Al}$ : 2,14; Si: 7,03; P: 9,76; Fe: 6,78; Mn: 3,27; Ti: 33,6; K: 1,99; Cl: $1 ; 04$.

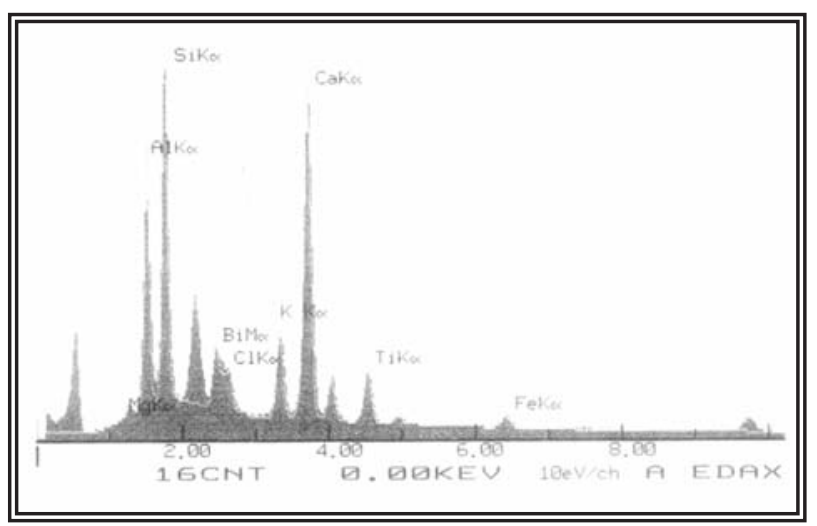

FIGURA 2. Contenido de iones (\% At). Al: 20,08; Si: 28,39; Bi: 2,21.

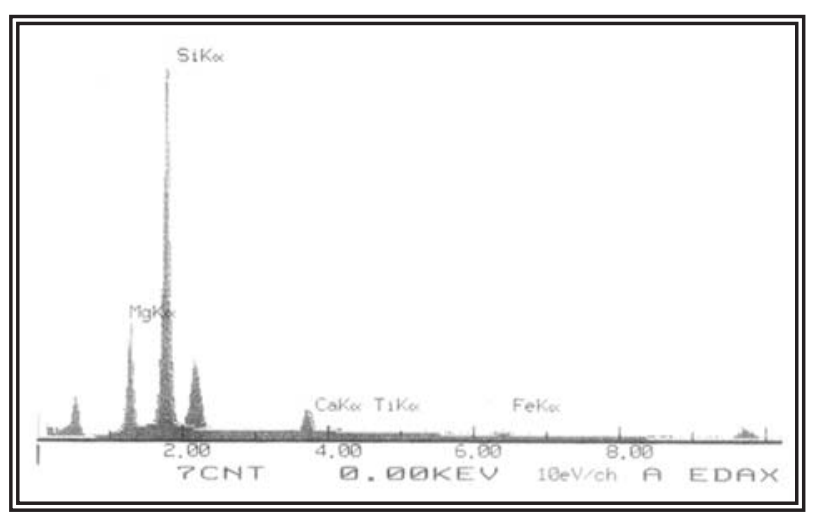

FIGURA 3. Contenido de iones (\% At). Mg: 24,69; Si: 68,23; Ti: 0,$34 ; \mathrm{Fe}: 0,88$; $\mathrm{Ca}: 5,81$.

medicamentos. En común, todos ellos presentan el hecho de eliminar una parte o su totalidad en la orina ${ }^{2,4}$.

Entre los oligoelementos se encuentran: el Zn, elemento implicado en los sistemas enzimáticos; el Fe que actúa en reacciones de óxido-reducción y el Mn que es constituyente de moléculas bioló-

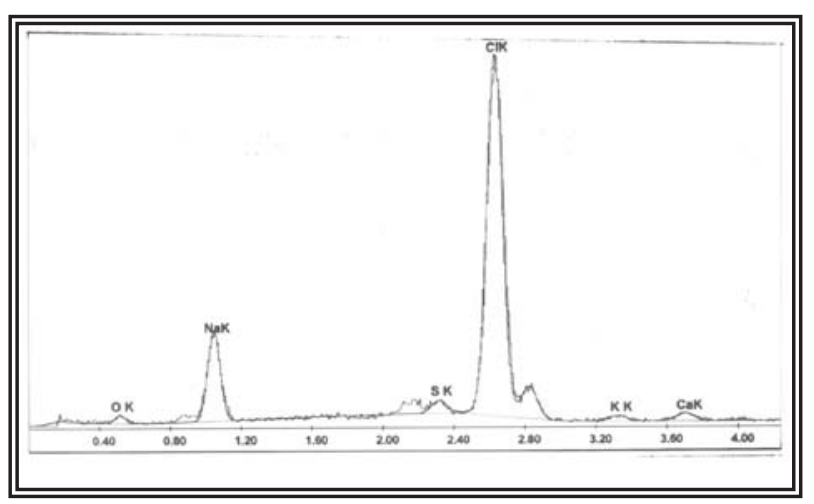

FIGURA 4. Contenido de iones (\% At). $\mathrm{Na:}$ 25,03; $\mathrm{Cl}: 53,6$.

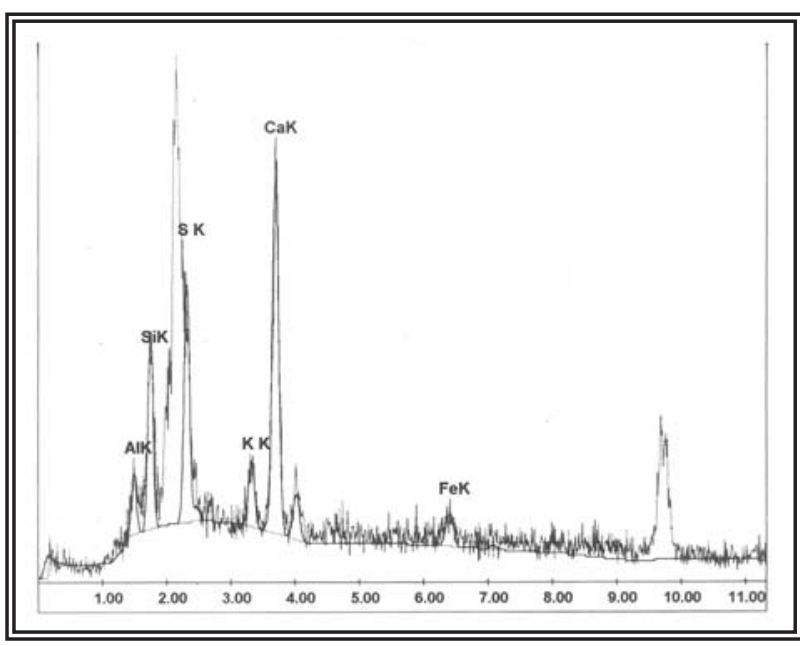

FIGURA 5. Contenido de iones (\% At). Al: 8,72; Si: 20,58, S: 24,04; K: 6,05; Ca: 35,5; Fe: 6,11.

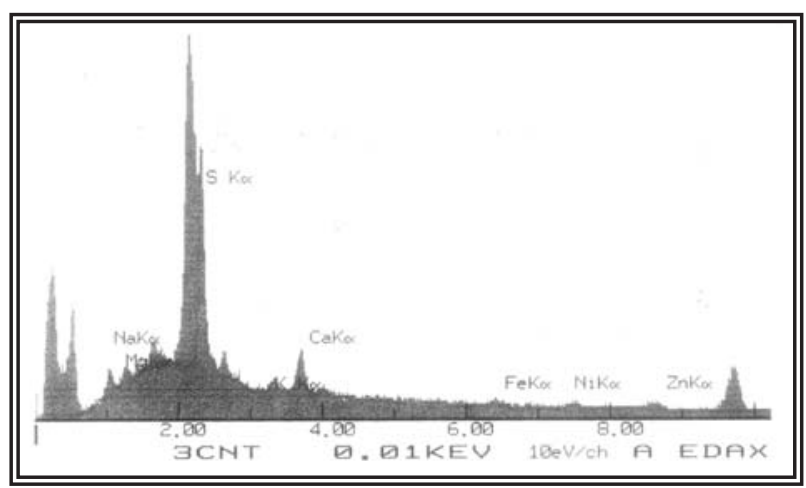

FIGURA 6. Contenido de iones (\% At). S: 61,43 ; Fe: 2,4 ; Ni: 2,04; Zn: 5,12 .

gicas, ofreciendo estabilidad a las estructuras tridimensionales. Estos elementos, en parte, pueden eliminarse en la orina.

El Si es un elemento que puede actuar en la calcificación del tejido conjuntivo. Un incremento en su captación repercute en un aumento de su eliminación urinaria. 


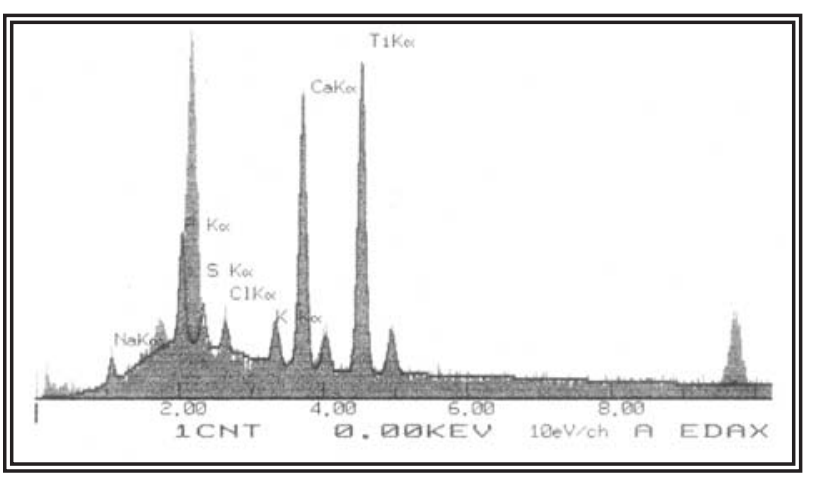

FIGURA 7. Contenido de iones (\% At). Ti: 46,79; Na: 10,78.

Aunque el $\mathrm{Al}$ es un elemento traza, su fuente principal proviene de fármacos orales, particularmente los destinados a reducir la hiperfosfatemia (sales intravenosas y dializadas).

$\mathrm{El} \mathrm{Ni}$ es otro elemento que se obtiene a través de la alimentación y es eliminado por el riñón.

$\mathrm{El}$ Na y el K se encuentran en sangre en forma de cloruros y son imprescindibles en la vida humana.

Por otra parte, el Ti se utiliza como excipiente en cremas, el $\mathrm{Zn}$ se encuentra en complejos vitamínicos, como también ocurre con el Sr; el Mn forma parte de sulfatos; el Ni de carbonatos; el $\mathrm{Si}$ forma parte de trisilicatos de $\mathrm{Al}$ (en tratamientos para dispepsias gastroduodenales); el S se utiliza en fármacos en forma de tiosulfato y el $\mathrm{Fe}$ en encuentra en las sales de Fe. El Bi está en forma de nitratos y salicilatos (componentes de la ranitidina utilizada como antiácido).

En un trabajo previo de nuestro grupo ${ }^{5}$ se encontró un cálculo renal que contenía $\mathrm{Si}, \mathrm{Al}, \mathrm{Fe}$, $\mathrm{S}$ y $\mathrm{Ba}$ aunque el componente fundamental del cálculo era whewellita. Según la historia clínica del paciente, la presencia de estos elementos era debida al tratamiento farmacológico que recibía.

El Si es un elemento poco frecuente en la especie humana. Sin embargo, algunas autores ya han descrito casos de cálculos cuya composición mayoritaria era $\mathrm{Si}^{6-9}$.

En el caso reportado por Rodríguez-Miñón et al. ${ }^{5}$ se describió que el Fe observado en el cálculo se debía a la ingesta de globulina férrica prescrita al paciente durante meses, se especuló que la presencia de Na y K era debida a la administración de sulfamidas.

Otro hecho descrito por nuestro equipo es el hallazgo de un cálculo eliminado compuesto por sulfato cálcico dihidrato ${ }^{10}$, analizado por espectroscopia infrarroja sin encontrar otros compo- nentes. Con posterioridad, se llevó a cabo un estudio por microscopia electrónica y EDAX haciendo patente su composición exclusiva de Ca y $\mathrm{S}$.

La descripción de cálculos de $\mathrm{SO}_{4} \mathrm{Ca}-2 \mathrm{H}_{2} \mathrm{O}$ es muy rara. Lagergren ${ }^{11}$ reconoció la existencia de sulfato cálcico mediante difracción de rayos $\mathrm{X}$ en dos cálculos compuestos fundamentalmente por ácido úrico. Hesse, et al. ${ }^{12}$ también describieron la existencia de sulfato cálcico en cuatro casos de 10.000 cálculos analizados.

El hecho de no encontrar elementos en la espectroscopia infrarroja es debido a que este sistema sólo detecta compuestos químicos y no elementos químicos. Esta observación hace necesario, en muchos casos, un estudio más exhaustivo de los cálculos complementándolo con otras técnicas como son las ya descritas.

Los resultados descritos en este trabajo son de gran relevancia y muestran que la diversidad de elementos eliminados por la orina son capaces de incorporarse a un cálculo para constituir parte del mismo o formar la totalidad del cálculo como el descrito por Cifuentes et $\mathrm{al}^{10}$.

\section{REFERENCIAS}

1. Rapado A, Álvarez-Arroyo MV, Traba ML. La litiasis renal urinaria como enfermedad metabólica. En: Litiasis urinarias. Ed A Rapado, JM Sulgdre, E Pérez-Castro, Ellendt 1991. Ediciones Doyma. Barcelona (pg 13-18).

2. Rapado A, Traba ML, Caycho C, Cifuentes-Delatte L. Drug-induced renal stones: incidence, clinical expression and stone analysis. Contrib Nephrol 1987;55:25-29.

3. Traba ML, De la Piedra C. El laboratorio en el estudio de la litiasis renal. Tiempos Médicos 1987;344:41-62.

4. Villa E, Navarro-Blasco I, Martín-Pérez A. Elementos traza. En: Tratado de Nutrición. Edición Díaz de Santos. Editor: Hernández Rodríguez, et al. 1999 (pg 229-247).

5. Rodríguez-Miñón JL, Salvador E, Bellameto J, Medina JA. Silica, Aluminium iron, sulfur and barium in a urinary calculus. Actas Urol Esp 1994;18:452-454.

6. Medina SA, Rodríguez J, Santos N, Cifuentes-Delatte L. Siliceous renal calculi in a man (11th case in the world). Rev Clin Esp 1978;151(5): 411-414.

7. Conte A, Montserrat V, Grasses F, Gutiérrez C, Coll R, García A. Litiasis urinaria de sílice. Actas Urol Esp 1989;13:405-407.

8. Yamamoto N, Maeda S, Shinoda I, Takeuchi T, Fujihiro S, Kanematsu M, Kuriyama M, Ban Y, Kawada Y. A case of silicate urolithiasis. Hinyokika Kiyo 1990;36(2):147-150.

9. Nishikawa Y, Ito H, Fuse H, Shimazaki J. Silica calculi, a case report Hinyokika-Kiyo 1991;37:625-627.

10. Cifuentes-Delatte L, Medina JA, Traba ML. A calcium sulfate calculus of renal origin. A verifiable observation?. Arch Esp Urol 1983;36:117-123.

11. Lagergren C. Biophysical investigations of urinary calculi. An x-ray crystallographic and microradiographic study. Acta Radiol 1956; Suppl 133:1-71.

12. Hess A, Schneider HJ, Schroder S, Vegner R. Results of analyses of 10.000 urinary calculi using electronic data processing methods. Zschr Urol 1976;69:1-9.

Dra. Maㅡ. Traba Villameytide

Laboratorio de Bioquímica

Fundación Jiménez Díaz-UTE

Avda. Reyes Católicos, 2 - 28040 Madrid

(Trabajo recibido el 10 de mayo 2005) 